\title{
Outcome of radioiodine therapy without, on or 3 days off carbimazole: a prospective interventional three-group comparison
}

\author{
Martin A. Walter ${ }^{1,}$, , Mirjam Christ-Crain², Christian Schindler ${ }^{3}$, Jan Müller-Brand ${ }^{1}$, Beat Müller ${ }^{2}$ \\ 1 Institute of Nuclear Medicine, University Hospital, Petersgraben 4, 4031 Basel, Switzerland \\ 2 Division of Endocrinology, Diabetology and Clinical Nutrition, University Hospital Basel, Basel, Switzerland \\ ${ }^{3}$ Institute of Social and Preventive Medicine, University Hospital Basel, Basel, Switzerland
}

Received: 26 November 2005 / Accepted: 2 February 2006 / Published online: 11 April 2006

(C) Springer-Verlag 2006

\begin{abstract}
Purpose: Carbimazole ameliorates hyperthyroidism but reduces radioiodine uptake and adversely affects the outcome of simultaneous radioiodine therapy. We explored whether withdrawal of carbimazole for 3 days can restore the outcome of radioiodine treatment without concurrent exacerbation of hyperthyroidism. By generating three groups with comparable radioiodine uptake, we also investigated whether the effect of carbimazole depends on the radioiodine uptake.

Methods: Stratified by a radioiodine uptake $>30 \%, 227$ consecutive adult patients were prospectively assigned to radioiodine therapy (target dose 200 Gy) without, on or 3 days off carbimazole. Patients were clinically (CrooksWayne score) and biochemically $\left(\mathrm{T}_{3}, \mathrm{fT}_{4}, \mathrm{TSH}\right)$ followed up after 3, 6 and 12 months. Primary endpoint was outcome 12 months after radioiodine therapy.

Results: A total of 207 patients completed follow-up (toxic nodular goitre, $n=117$; Graves' disease, $n=90$ ). The overall success rate was $71.5 \%$. Patients without and 3 days off carbimazole had similar biochemical $(81.4 \%$ and $83.3 \%$, respectively; $p=0.82$ ) and clinical outcomes [median (range) Crooks-Wayne score $0(0-16)$ and $1(0-10)$, respectively; $p=0.73]$, which were both higher than in patients on carbimazole $[42.6 \%, p<0.001$; Crooks-Wayne score $3(0-30), p<0.03]$. Time to achieve cure was delayed on carbimazole. No changes in thyroid hormone levels occurred after 3 days' discontinuation of carbimazole. Logistic regression revealed that all observed cure rates were independent of entity, sex, age, thyroid volume, radioiodine uptake, radioiodine half-life, $\mathrm{fT}_{4}, \mathrm{~T}_{3}$ and $\mathrm{TSH}$. Conclusion: Patients under carbimazole treatment can be referred for radioiodine therapy after withdrawal of
\end{abstract}

Martin A. Walter ( $)$

Institute of Nuclear Medicine,

University Hospital,

Petersgraben 4,

4031 Basel, Switzerland

e-mail: m.a.walter@gmx.net

Tel.: +41-61-2655684, Fax: +41-61-2654897 carbimazole for only 3 days. Three days of carbimazole withdrawal is long enough to restore the success of radioiodine therapy and short enough to avoid the risk of exacerbation of hyperthyroidism.

Keywords: Radioiodine - Hyperthyroidism - Graves' disease - Toxic nodular goitre - Carbimazole -

Anti-thyroid drugs

Eur J Null Med Mol Imaging (2006) 33:730-737

DOI 10.1007/s00259-006-0092-8

\section{Introduction}

Hyperthyroid patients admitted to radioiodine therapy are often prescribed carbimazole to accelerate the return of euthyroidism, to ameliorate clinical symptoms and to prevent the exacerbation of hyperthyroidism after administration of radioiodine [1, 2]. Unfortunately, patients receiving radioiodine under simultaneous carbimazole medication show significantly lower success rates than patients who do not receive carbimazole [3-5]. Withdrawal of carbimazole has the potential to overcome this detrimental effect [6-8], but discontinuation for 5 days or longer leads to exacerbation of hyperthyroidism [9]. Reports from prospective interventional studies on the outcome of radioiodine treatment after a brief period of carbimazole withdrawal, however, are limited $[5,7,8]$.

The underlying mechanism of the detrimental effects of carbimazole on the success rate of radioiodine treatment is not fully understood. Carbimazole decreases the peroxidase-catalysed synthesis of oxygen free radicals, which mediate the cell damage produced by radioiodine $[10,11]$. On the other hand, carbimazole reduces the cellular radioiodine uptake $[3,5]$, which has also been thought to decrease the success of radioiodine therapy [12, 13]. Accordingly, low success of radioiodine therapy under 
carbimazole has only been described in conjunction with significantly lower radioiodine uptake under carbimazole $[3,5]$.

Against this background, our first aim was to explore whether 3 days of carbimazole withdrawal can fully restore the success of radioiodine treatment without concurrent exacerbation of hyperthyroidism. Second, we aimed to explore whether carbimazole impairs the outcome of radioiodine therapy independently of the radioiodine uptake; for this purpose we generated three patient groups (without, on and 3 days off carbimazole) with comparable radioiodine uptake.

\section{Materials and methods}

\section{Inclusion criteria}

Patients older than 18 years with confirmed hyperthyroidism due to Graves' disease or toxic nodular goitre were eligible for inclusion (Fig. 1). The diagnosis of Graves' disease was based on suppressed serum TSH levels $(<0.25 \mathrm{mIU} / \mathrm{l})$, morphology on ultrasound, scintigraphic confirmation of diffuse goitre and elevated serum TSH receptor antibodies. The diagnosis of toxic nodular goitre was based on suppressed serum TSH levels $(<0.25 \mathrm{mIU} / \mathrm{l})$, morphology on ultrasound and scintigraphic confirmation of thyroid nodules. Exclusion criteria were pretreatment with antithyroid drugs other than carbimazole, pregnancy, breast-feeding or incontinence. Radioiodine therapy was not performed when radioiodine uptake was lower than $30 \%$, as uptake below this level has been shown to significantly decrease the success of radioiodine treatment [12]. The population in the referral area has a negligible incidence of dietary iodine deficiency owing to mandatory iodine supplementation of dietary salt in Switzerland. The study was approved by the local ethics committee (approval no. EKBB145/02), informed consent was obtained and the endpoints were predefined.

Generation of patient groups without, on and 3 days off carbimazole with comparable radioiodine uptake

The three groups were stratified as follows: Radioiodine uptake was measured in all patients. Patients pretreated with carbimazole (by their general practitioner or endocrinologists) who had radioiodine uptake exceeding $30 \%$ were assigned to the group on carbimazole. Thereby, radioiodine therapy was performed under simultaneous carbimazole treatment. On the other hand, if the radioiodine uptake was below $30 \%$, carbimazole was discontinued for 3 days prior to radioiodine treatment, a measure which, in our experience, significantly increases the radioiodine uptake [14]. The third group comprised who were not treated with carbimazole, i.e. were assigned to receive radioiodine treatment without carbimazole.

\section{Biochemical investigation}

Thyroid function was assessed by measuring serum free $\mathrm{T}_{4}$ (RIAgnost FT4, CIS bio international, reference range 9.0-24.5 pmol/1), serum $\mathrm{T}_{3}$ (RIA-gnost T3, CIS bio international, reference range 0.9 $2.5 \mathrm{nmol} / \mathrm{l}$ ) and serum TSH (RIA-gnost TSH, CIS bio international, reference range $0.25-4.0 \mathrm{mIU} / \mathrm{l})$. Hyperthyroidism was defined as an abnormally low serum TSH level, while hypothyroidism was defined as a TSH level above the reference range. Subclinical hyperthyroidism was defined as a serum TSH level below the normal reference range and serum $\mathrm{fT}_{4}$ and $\mathrm{T}_{3}$ levels within the respective reference ranges [15].

\section{Iodine kinetics and thyroid scintigraphy}

Iodine kinetics were determined as previously described $[14,16]$. In brief, thyroid uptake was measured at 2, 4, 24 and $48 \mathrm{~h}$ after application of $7 \mathrm{MBq}{ }^{123} \mathrm{I}$ using a previously calibrated scintillation counter with a 3-inch sodium iodide crystal and scalar ratemeter (Biodex Medical Systems). Measurement was performed for 2 min with the patient positioned upright and with the anterior surface of the neck $25 \mathrm{~cm}$ from the detector. Attenuation correction was done with the detector shielded from the remainder of the body. Resulting uptake (decay and dead time corrected) was calculated using background radiation correction and reference standard: thyroid uptake $=$ (neck counts - thigh counts $) /($ reference counts - room counts $)$. The peak radioiodine uptake assessed at $2,4,24$ or $48 \mathrm{~h}$ was employed to define the radioiodine uptake for stratification and subsequent dose calculation. Scintigraphy was performed using a gamma camera equipped with a low-energy high-resolution parallelhole collimator (Basicam, Siemens, Erlangen, Germany). Each image was acquired for $10 \mathrm{~min}$. The images were interpreted using both computer screen (256 grey scale) and film by board-certified nuclear medicine physicians.

\section{Thyroid ultrasound}

Ultrasound was performed for determination of the thyroid volume using a Logiq $400 \mathrm{MD}$ and a Kodak Laser Imager Keypad transducer. Volume was determined by an ellipsoid model (mass= height $\times$ width $\times$ depth $\times 0.52$ ) of each lobe and the height, width and depth of the isthmus [17].

\section{Radioiodine therapy}

Radioiodine treatment was performed as previously described [16]. The individual activity applied was calculated according to Marinelli's formula [18]: Dose of ${ }^{131} \mathrm{I}(\mathrm{MBq})=$ Intended dose $(\mathrm{Gy}) \times$ Target volume $(\mathrm{ml}) \times 25 /$ Maximal radioiodine uptake $(\%) \times T_{1 / 2}(\mathrm{~h})$. Intended tissue-absorbed doses of $200 \mathrm{~Gy}$ were administered. Patients in whom carbimazole had been withdrawn for 3 days resumed carbimazole medication 4 days after radioiodine administration. Thyrostatic medication was discontinued in all patients 6 weeks after radioiodine treatment. Radioiodine therapy was accompanied by the provision of standardised radiation protection guidelines and information that hypothyroidism was a likely outcome of radioiodine therapy. 
Follow-up

Patients were initially examined and followed up at 3,6 and 12 months, by clinical assessment using the semiquantitative CrooksWayne score [19]. This score includes 13 discriminating signs and symptoms of hyperthyroidism and ranges between 0 and 31 points, with higher values indicating more severe clinical hyperthyroidism. Furthermore, patients were assessed by measuring serum $\mathrm{T}_{3}, \mathrm{fT}_{4}$ and TSH levels at baseline and 3, 6 and 12 months after radioiodine treatment. Successful radioiodine therapy was defined by clinical and biochemical evidence of euthyroidism or hypothyroidism at 12 months after radioiodine treatment.

\section{Statistical analysis}

Discrete variables are expressed as counts (percentage) and continuous variables as median (range), unless otherwise stated. The endpoints were predefined. Frequency comparison was performed by means of the chi-square test. Kruskal-Wallis one-way analysis of variance with Dunn's multiple post hoc test was used if more than two groups were being compared. The statistical significance of changes in quantitative measures over time was assessed using the signed rank test. To test whether differences in success rates between groups persisted when taking into account differences in patients' baseline characteristics across groups, we performed a logistic regression analysis with treatment success as the binary outcome variable and sex, age, thyroid volume, radioiodine uptake, radioiodine half-life, $\mathrm{fT}_{4}, \mathrm{~T}_{3}, \mathrm{TSH}$, baseline Crooks-Wayne score and administered radioiodine dose as covariates. Backward selection with a threshold significance level of 0.2 was then applied to obtain a more parsimonious model. A $p$ value of $<0.05$ was considered to be statistically significant. Data were analysed using Statistica V.6.0 for Windows (StatSoft, Inc., Tulsa, OK, USA).

\section{Results}

Study groups without, on or 3 days off carbimazole

Of 262 consecutive patients who were transferred for radioiodine treatment between 1 August 2002 and 1 August 2004, 259 (98.9\%) were eligible for inclusion. Thirty-two patients were excluded owing to low radioiodine uptake and 227 patients were prospectively assigned to receive radioiodine treatment without, on or 3 days off carbimazole (Fig. 1). Five patients (four females, one male; median age 78 years, range 68-86 years) died in the first year after radioiodine treatment without revealing clinical signs of exacerbation of hyperthyroidism. Fifteen patients were lost to follow-up, leaving 207 patients for final analysis. The baseline criteria of the 20 lost patients did not differ significantly from those of the remaining cohort (data not shown).

Based on the study design, three groups without ( $n=129 ; 88$ toxic nodular goitre patients, 41 Graves' disease patients), on ( $n=54 ; 18$ toxic nodular goitre patients, 36 Graves' disease patients) and 3 days off ( $n=24$; 11 toxic nodular goitre patients, 13 Graves' disease patients) carbimazole and with comparable radioiodine uptake values were generated (Table 1). The three groups were also well balanced regarding sex, age, goitre volume, radioiodine half-life, baseline Crooks-Wayne score, serum $\mathrm{T}_{3}$ level and the percentage of patients with subclinical

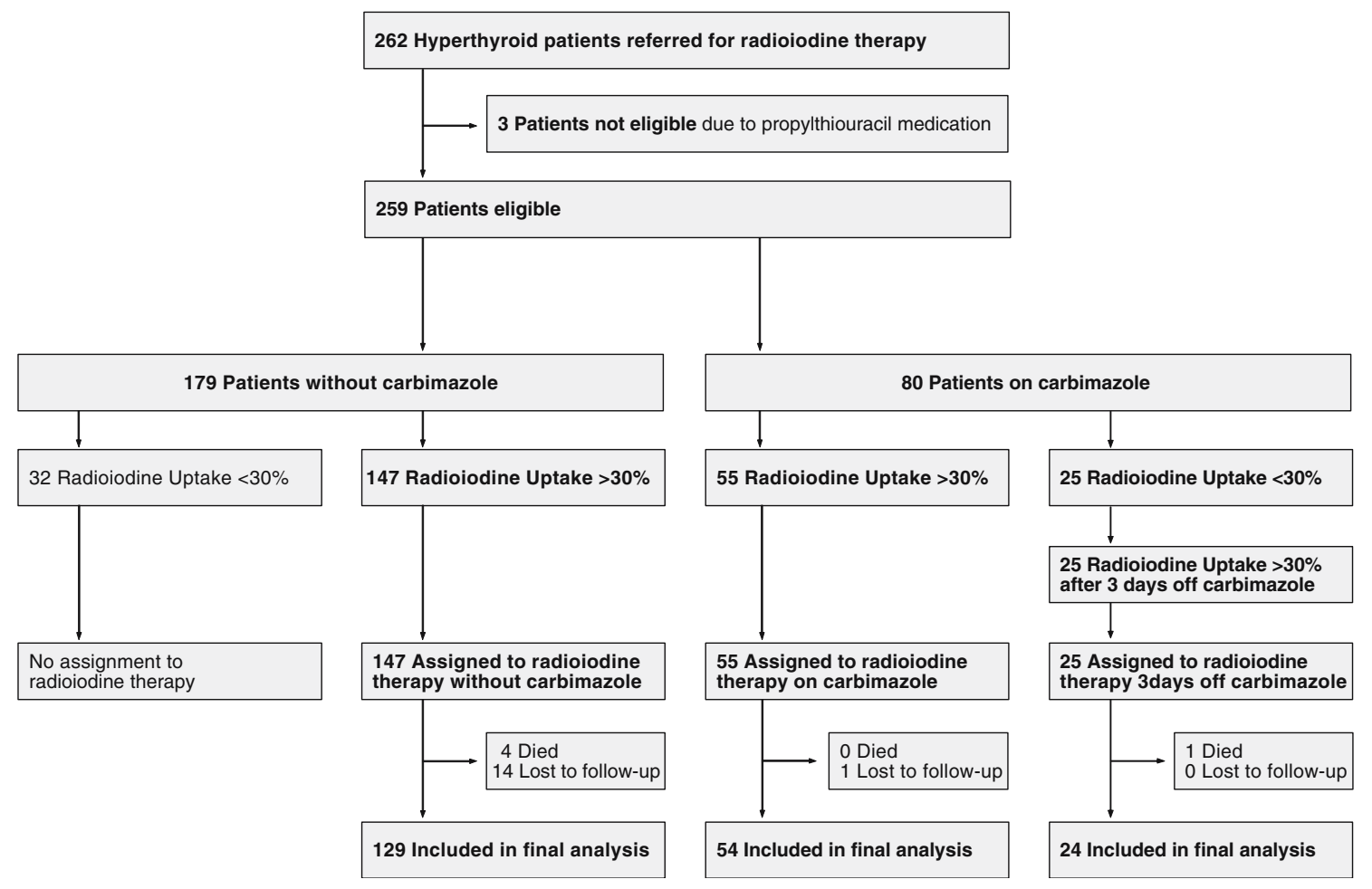

Fig. 1. Trial profile 
hyperthyroidism. As expected, patients in the group without carbimazole had higher $\mathrm{fT}_{4}$ and lower TSH levels than patients in the on and 3 days off carbimazole groups. Based on Marinelli's formula, higher radioiodine activities were administered to patients on carbimazole to achieve target doses comparable to those in patients without and 3 days off carbimazole.

One year outcome without, on or 3 days off carbimazole

Overall, radioiodine treatment resulted in elimination of biochemical hyperthyroidism in $71.5 \%(148 / 207)$ of all patients: euthyroidism was achieved in $43.5 \%(90 / 207)$ and hypothyroidism in $28.0 \%(58 / 207)$. Subgroup analysis revealed that patients receiving radioiodine without carbimazole showed a success rate of $81.4 \%(105 / 129$; $95 \%$ confidence interval 73.6-87.7\%), while patients on carbimazole had a success rate of $42.6 \%(23 / 54 ; 95 \%$ confidence interval $29.2-56.8 \% ; p<0.001)$. Patients in whom carbimazole was withdrawn for 3 days had a success rate of $83.3 \%(20 / 24 ; 95 \%$ confidence interval $62.6-$ $95.3 \%$ ), which was significantly higher than the success rate on carbimazole $(p<0.0001)$ and comparable to the success of radioiodine treatment without carbimazole ( $p=0.82$, Fig. 2a).

The pretherapeutic Crooks-Wayne scores of patients without (median 7, range 0-28), on (median 8, range 0-26) and 3 days off carbimazole (median 6, range 0-27) were comparable ( $p=0.17$, Table 1$)$. In contrast, 12 months after radioiodine treatment the Crooks-Wayne score was significantly higher in patients on carbimazole (median 3, range $0-30$ ) than in patients without (median 0 , range 0 $16)$ or 3 days off carbimazole (median 1, range $0-10$ ) $(p=0.024$, Fig. 2b).
Subgroup analysis of the biochemical outcome confirmed the overall results in patients suffering from toxic nodular goitre as well as in patients suffering from Graves' disease. In toxic nodular goitre patients $(n=117)$, the 1-year success rate on carbimazole $(50.0 \%)$ was significantly lower than that without carbimazole $(86.4 \%, p<0.001)$ or 3 days off carbimazole $(90.9 \%, p=0.025)$, while the outcome without and 3 days off carbimazole was similar ( $p=0.67$, Fig. 3a). In patients suffering from Graves' disease $(n=90)$, the success rate was also significantly lower in patients on carbimazole (38.9\%) than in patients without carbimazole $(70.7 \%, p=0.005)$ or 3 days off carbimazole $(76.9 \%, p=0.019)$, whereas the success rates without and 3 days off carbimazole were similar $(p=0.66$, Fig. 3b).

Logistic regression analysis revealed that the unadjusted odds ratio of a successful radioiodine therapy between patients without and patients on carbimazole was 0.21 (95\% confidence interval $0.10-0.41, p<0.0001$ ). In contrast, the corresponding odds ratio comparing patients without and patients 3 days off carbimazole was 1.39 (95\% confidence interval $0.44-4.4, p=0.58$ ). The covariateadjusted odds ratio (adjusted for sex, age, thyroid volume, radioiodine uptake, radioiodine half-life, $\mathrm{fT}_{4}, \mathrm{~T}_{3}, \mathrm{TSH}$, Crooks-Wayne score and administered radioiodine dose, respectively) of a successful radioiodine therapy comparing patients without and patients on carbimazole was 0.13 (95\% confidence interval $0.04-0.37, p<0.0001$ ). In contrast, the corresponding odds ratio comparing patients without and patients 3 days off carbimazole was 1.35 (95\% confidence interval $0.25-7.3, p=0.72$ ).

Table 1. Baseline investigation results $(n=207)$

\begin{tabular}{|c|c|c|c|c|}
\hline & $\begin{array}{l}\text { Off carbimazole } \\
(n=129)\end{array}$ & $\begin{array}{l}\text { On carbimazole } \\
(n=54)\end{array}$ & $\begin{array}{l}3 \text { Days off carbimazole } \\
(n=24)\end{array}$ & $p$ value \\
\hline Females (\%) & 85.3 & 72.2 & 79.2 & n.s. \\
\hline Age (years) & $67(25-90)$ & $60(25-83)$ & $69(28-87)$ & n.s. \\
\hline Goitre volume $(\mathrm{ml})$ & $27.2(7.6-264.8)$ & $34.5(8.0-560.0)$ & $25.8(8.0-97.0)$ & n.s. \\
\hline Radioiodine uptake (\%) & $46.7(31.0-88.8)$ & $50.3(30.0-92.6)$ & $57.3(32.8-81.2)^{\mathrm{a}}$ & n.s. \\
\hline Radioiodine half-life (days) & $5.5(0.6-7.9)$ & $5.5(0.8-7.4)$ & $5.5(2.1-6.8)$ & n.s. \\
\hline Crooks-Wayne score (points) & $7(0-28)$ & $8(0-26)$ & $6(0-27)$ & n.s. \\
\hline $\mathrm{fT}_{4}(\mathrm{pmol} / \mathrm{l})^{\mathrm{b}}$ & $22.9(11.5-86.2)$ & $15.4(2.3-73.3)$ & $18.7(10.5-63.5)$ & $=0.001$ \\
\hline $\mathrm{T}_{3}(\mathrm{nmol} / \mathrm{l})^{\mathrm{b}}$ & $2.5(1.3-7.3)$ & $2.3(1.3-6.6)$ & $2.2(1.0-9.1)$ & n.s. \\
\hline $\mathrm{TSH}(\mathrm{mIU} / \mathrm{l})^{\mathrm{b}}$ & $0.03(0.004-0.19)$ & $0.08(0.03-14.0)$ & $0.03(0.03-1.5)$ & $<0.001$ \\
\hline Subclinical hyperthyroidism (\%) & 61.2 & 46.3 & 45.8 & n.s. \\
\hline Radioiodine activity $(\mathrm{MBq})^{\mathrm{c}}$ & $444(185-814)$ & $518(259-1,295)$ & $481(222-925)$ & n.s. \\
\hline
\end{tabular}

Values are expressed as median (range)

n.s. non-significant

a Radioiodine uptake values after 3 days of withdrawal of carbimazole

b Reference ranges are: $\mathrm{fT}_{4}, 9.0-24.5 \mathrm{pmol} / 1 ; \mathrm{fT}_{3}, 0.9-2.5 \mathrm{nmol} / \mathrm{l} ; \mathrm{TSH}, 0.25-4.0 \mathrm{mIU} / 1$

${ }^{\mathrm{c}}$ Megabequerels can be converted to microcuries by dividing by 37 


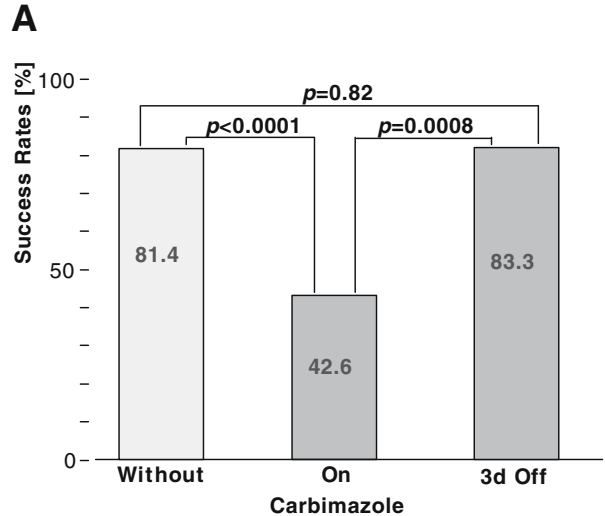

Fig. 2. Overall biochemical (a) and clinical (b) outcome of radioiodine treatment without, on or 3 days off carbimazole after 12 months. a Biochemical success rate (\%) in patients receiving radioiodine treatment on simultaneous carbimazole medication, compared with the success rate in patients without carbimazole or

\section{Radioiodine uptake and biochemical thyroid status on and 3 days off carbimazole}

In the group of patients receiving radioiodine 3 days off carbimazole $(n=24)$, radioiodine uptake levels were measured both on and off carbimazole. Thereby, the radioiodine uptake was found to increase significantly $(p<0.001)$ from a median of $14.9 \%$ (range $4.5-25.4 \%$ ) on carbimazole to a median of $57.3 \%$ (range $32.8-81.2 \%$ ) 3 days off carbimazole. Hence, the median increase in radioiodine uptake was 3.8-fold (range 1.9-8.0 fold). On the other hand, there was no significant change in the effective halflife on carbimazole (median 5.3 days, range 0.8-7.3 days) and 3 days off carbimazole (median 5.5 days, range 2.1$6.8, p=0.12$ ).

The biochemical thyroid status was also measured on and off carbimazole in patients receiving radioiodine 3 days off carbimazole ( $n=24$, Fig. 4a). There were no significant differences between the levels of $\mathrm{fT}_{4}$

A

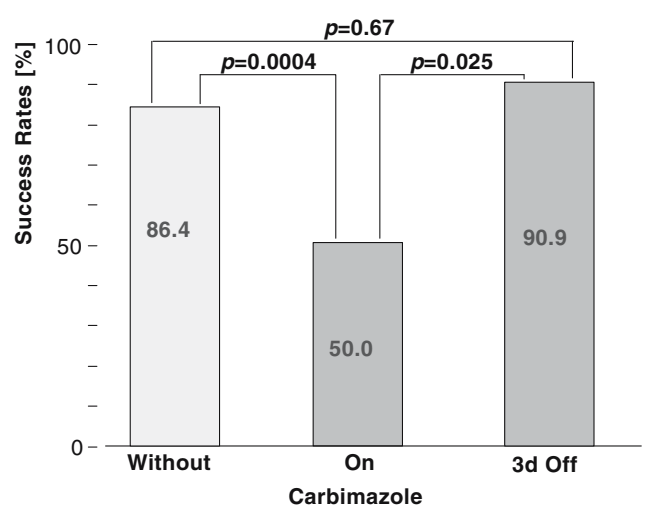

Fig. 3. Subgroup analysis: 1-year outcome of radioiodine therapy without, on or 3 days off carbimazole in patients suffering from toxic nodular goitre or Graves' disease. The biochemical 1-year success rate of radioiodine treatment $(\%)$ is shown in patients on

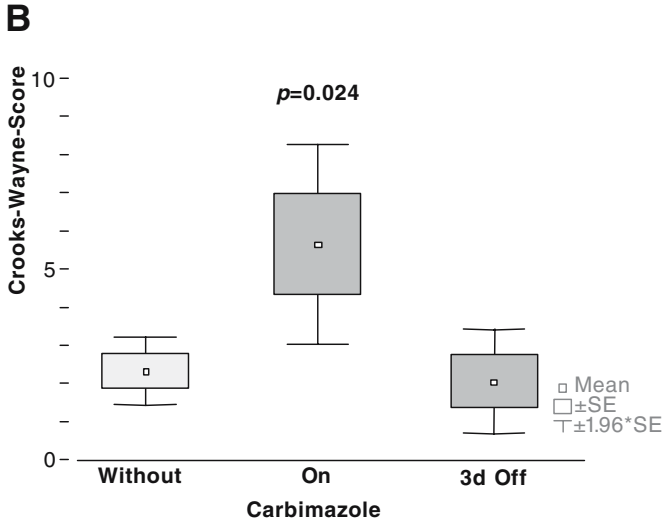

3 days off carbimazole, as assessed by serum TSH measurements. b Clinical success rate assessed by the Crooks-Wayne hyperthyroidism score in patients receiving radioiodine treatment on carbimazole, compared with the score in patients without carbimazole or 3 days off carbimazole

(median $18.7 \mathrm{pmol} / \mathrm{l}$, range $10.5-63.5 \mathrm{pmol} / \mathrm{l}$ ), $\mathrm{T}_{3}$ (median $2.2 \mathrm{nmol} / \mathrm{l}$, range $1.0-9.1 \mathrm{nmol} / \mathrm{l}$ ) and $\mathrm{TSH}$ (median $0.03 \mathrm{mIU} / \mathrm{l}$, range $0.03-1.50 \mathrm{mIU} / \mathrm{l}$ ) on carbimazole and the levels of $\mathrm{fT}_{4}$ (median $17.7 \mathrm{pmol} / \mathrm{l}$, range 9.7-42.8 pmol, $p=0.27$ ), $\mathrm{T}_{3}$ (median $2.4 \mathrm{nmol} / \mathrm{l}$, range $1.3-4.4$ $\mathrm{nmol} / 1, p=0.48$ ) and TSH (median $0.03 \mathrm{mIU} / 1$, range 0.03 $1.17 \mathrm{mIU} / 1, p=0.49) 3$ days off carbimazole. Additionally, no patient suffered from clinical aggravation of hyperthyroidism as a result of the 3-day carbimazole withdrawal.

Time to achieve euthyroidism after radioiodine therapy off, on or 3 days off carbimazole

Overall, hyperthyroidism was present in $45.6 \%$ of all patients after 3 months, $32.0 \%$ after 6 months and $28.5 \%$ after 12 months. At 3 months' follow-up, hyperthyroidism was present in $59.2 \%$ of patients who received radioiodine on carbimazole as compared to $44.3 \%$ without carbimazole

B

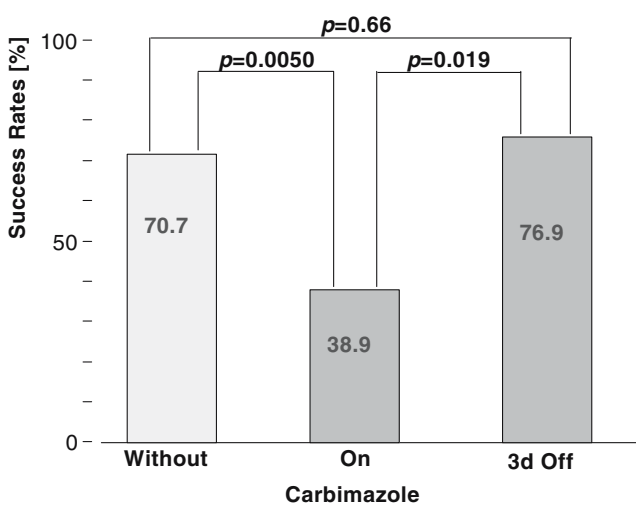

simultaneous carbimazole medication as compared to patients without carbimazole or 3 days off carbimazole: a in patients suffering from toxic nodular goitre $(n=117)$ and $\mathbf{b}$ in patients suffering from Graves' disease $(n=90)$ 
A

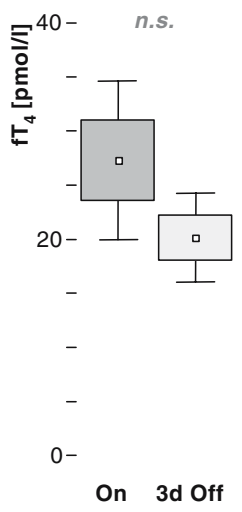

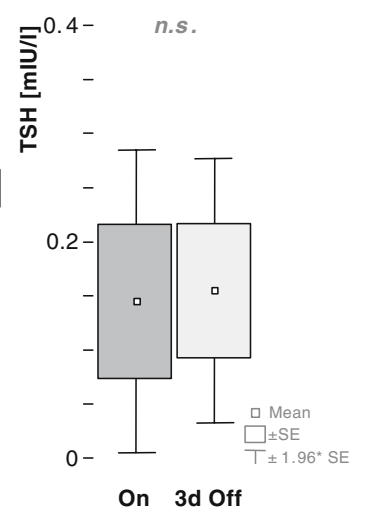

B

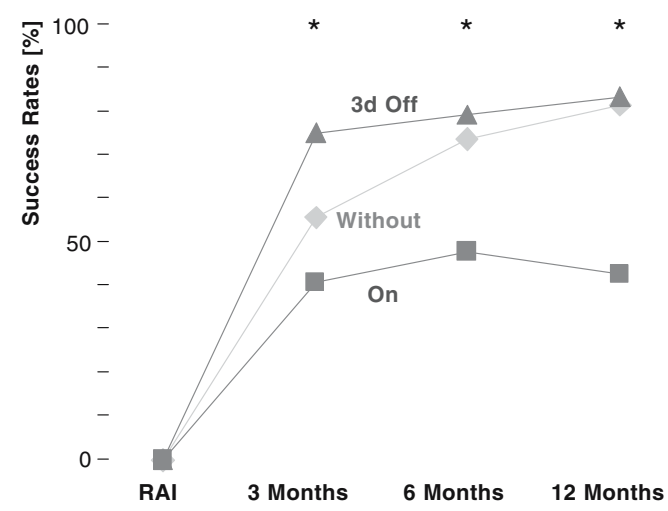

Fig. 4. The effect of 3 days' carbimazole withdrawal on the biochemical thyroid status (a) and the cure rate at different times after radioiodine therapy (b). a Levels of $\mathrm{fT}_{4}, \mathrm{~T}_{3}$ and $\mathrm{TSH}$ are shown on and off carbimazole ( $p=$ n.s.) in the subgroup with 3-day carbimazole withdrawal $(n=24)$. b The success rates after radioio-

and $25.0 \% 3$ days off carbimazole $(p=0.02)$. Six months after radioiodine treatment, hyperthyroidism was found in $52.4 \%$ of patients treated with radioiodine on carbimazole as compared to $26.4 \%$ in patients treated without carbimazole and $20.8 \%$ in patients treated 3 days off carbimazole $(p=0.004$, Fig. $4 \mathrm{~b})$.

\section{Discussion}

Based on our data, it can be asserted that simultaneous carbimazole medication reduces the success of radioiodine therapy in toxic nodular goitre and Graves' disease independently of the radioiodine uptake. Importantly, a 3-day withdrawal is long enough to overcome this effect and to restore the success of radioiodine treatment without the risk of exacerbating hyperthyroidism. Furthermore, a 3-day carbimazole withdrawal is able to reduce the time required to achieve cure after radioiodine therapy as compared with radioiodine treatment under carbimazole.

The cure rates of radioiodine therapy under adjuvant thyrostatic medication have been investigated by previous studies. However, most studies have merged patients on diverse antithyroid drugs, namely thiamazoles and thiouracils, which have distinct detrimental effects on radioiodine treatment [20]. The influence of carbimazole alone on radioiodine treatment has been investigated in only a few reports [3-8], in which low success rates of radioiodine therapy with concomitant carbimazole treatment have exclusively been associated with significantly lower radioiodine uptake under carbimazole [3,5]. Generally, low radioiodine uptake is assumed to adversely affect the success of radioiodine therapy [12, 13], although the opposite effect has also been reported [16]. It is to be noted, however, that in the above-mentioned reports, carbimazole was identified as a confounder of radioiodine treatment only in patient groups with incomparable baseline characteristics due to differences in radioiodine uptake. In dine treatment are shown in patients receiving radioiodine treatment on carbimazole medication compared to patients receiving radioiodine treatment without or 3 days off carbimazole, after 3 months $(p=0.0199), 6$ months $(p=0.0043)$ and 12 months $(p<0.0001)$ $(n=207)$

other words, no studies have previously addressed the question of whether carbimazole may exert an uptakeindependent detrimental effect on treatment outcome by investigating groups with comparable radioiodine uptake.

To answer this question, our study protocol stratified patients into groups with comparable radioiodine uptake. We found that, despite similar uptake of the isotope, patients on carbimazole had a significantly worse outcome than patients who did not receive carbimazole. Thus, simultaneous carbimazole medication was directly verified to be an independent confounder of radioiodine treatment. In addition, the low success rate of radioiodine therapy under simultaneous carbimazole therapy in spite of comparable radioiodine uptake strongly argues against a radioiodine uptake-mediated detrimental mechanism of carbimazole. In this context, radioprotection by the inhibition of peroxidase-catalysed synthesis of oxygen free radicals, which mediate the cell damage in radioiodine therapy $[10,11]$, appears to be a more likely mechanism.

Anti-thyroid drugs are routinely used in the management of both Graves' disease and toxic nodular goitre [2, $21,22]$. There is controversy over whether their effect on radioiodine treatment is similar in both diseases. Thiamazoles and thiouracils have been found to reduce the success of radioiodine treatment in Graves' disease [3, 5, 23-26]. In contrast, studies including both patients with Graves' disease and patients with toxic nodular goitre revealed propylthiouracil or multiple thyrostatics to have no effect in Graves' disease but an adverse influence in toxic nodular goitre $[21,22]$. However, the influence of simultaneous carbimazole medication alone on radioiodine therapy in toxic nodular goitre has not yet been investigated. The present study included patients with Graves' disease and patients with toxic nodular goitre. The overall negative effect of simultaneous carbimazole on radioiodine treatment was confirmed to be similar in both subgroups. Moreover, in contrast to previous reports, the negative effect of carbimazole on radioiodine therapy was con- 
firmed not only biochemically but also clinically, as quantified by the Crooks-Wayne score.

Previous reports have indicated that the success of radioiodine treatment is not diminished if carbimazole is discontinued for 16 days [6], 5 days [7] or 5 to 3 days [8] before administration of radioiodine. However, even merely 5 days of withdrawal can lead to aggravation of hyperthyroidism [9], which is especially of concern in patients with severe concomitant diseases. Our data indicated that a 3-day withdrawal of carbimazole restored the success of radioiodine therapy without an increase in peripheral thyroid hormones or clinical aggravation of hyperthyroidism. Nevertheless, this study did not have the statistical power to proof non-inferiority regarding treatment failure after 1 year.

Exacerbation of hyperthyroidism after radioiodine therapy should also be avoided. Consequently, radioiodine treatment is often performed under simultaneous carbimazole medication to prevent the increase in thyroid hormone levels that is frequently observed in the first weeks after radioiodine therapy without adjuvant thyrostatic medication [27]. However, contrary to this intended effect, the present study revealed a significant delay in the return to euthyroidism if radioiodine was performed under carbimazole. Nevertheless, 3 days of carbimazole withdrawal could overcome this effect.

Our study has limitations. The patient groups without, on and 3 days off carbimazole were imbalanced with regard to the number of patients. However, this imbalance is a consequence of the study protocol, which had the primary aim of generating groups with comparable radioiodine uptake. Despite the limited number of patients receiving radioiodine 3 days off carbimazole, we observed highly significant detrimental effects of simultaneous carbimazole therapy. One might also argue that a selection bias occurred as a result of arranging groups according to their treatment at the time of inclusion. However, in view of the similar baseline characteristics of the three groups, this is unlikely to have resulted in significant bias. Furthermore, if randomisation is used to prevent a potential selection bias, a systemic bias occurs owing to lower radioiodine uptake in patients under carbimazole $[3,5]$. Our therapy results were derived from patients with radioiodine uptake exceeding 30\%. Further prospective interventional studies would be helpful in clarifying the effect of carbimazole on radioiodine treatment in patients with lower radioiodine uptake.

In conclusion, patients under carbimazole treatment can be referred for radioiodine therapy after withdrawal of carbimazole for only 3 days. Three days of carbimazole withdrawal is long enough to restore the success of radioiodine therapy and short enough to avoid the risk of exacerbation of hyperthyroidism.

\section{References}

1. Solomon B, Glinoer D, Lagasse R, Wartofsky L. Current trends in the management of Graves' disease. J Clin Endocrinol Metab 1990;70:1518-1524

2. Cooper DS. Antithyroid drugs. N Engl J Med 2005;352:905-917

3. Clerc J, Izembart M, Dagousset F, Jais JP, Heshmati HM, Chevalier A, et al. Influence of dose selection on absorbed dose profiles in radioiodine treatment of diffuse toxic goiters in patients receiving or not receiving carbimazole. J Nucl Med 1993;34:387-393

4. Sabri O, Zimny M, Schreckenberger M, Reinartz P, Ostwald E, Buell U. Radioiodine therapy in Graves' disease patients with large diffuse goiters treated with or without carbimazole at the time of radioiodine therapy. Thyroid 1999;9:1181-1188

5. Sabri O, Zimny M, Schulz G, Schreckenberger M, Reinartz P, Willmes K, et al. Success rate of radioiodine therapy in Graves' disease: the influence of thyrostatic medication. J Clin Endocrinol Metab 1999;84:1229-1233

6. Razvi S, Basu A, McIntyre EA, Wahid ST, Bartholomew PH, Weaver JU. Low failure rate of fixed administered activity of $400 \mathrm{MBq}{ }^{131}$ I with pre-treatment with carbimazole for thyrotoxicosis: the Gateshead Protocol. Nucl Med Commun 2004;25:675-682

7. Connell JM, Hilditch TE, Robertson J, Coghill G, Alexander WD. Radioprotective action of carbimazole in radioiodine therapy for thyrotoxicosis - influence of the drug on iodine kinetics. Eur J Nucl Med 1987;13:358-361

8. Goolden AW, Fraser TR. Effect of pretreatment with carbimazole in patients with thyrotoxicosis subsequently treated with radioactive iodine. Br Med J 1969;3:443-444

9. Burch HB, Solomon BL, Wartofsky L, Burman KD. Discontinuing antithyroid drug therapy before ablation with radioiodine in Graves disease. Ann Intern Med 1994;121:553-559

10. Wilson R, McKillop JH, Buchanan LM, Bradley H, Smith WE, Thomson JA. The effect of carbimazole therapy on interleukin 2 , interleukin 2 receptors and free radicals. Autoimmunity 1990;8:3-7

11. Abalovich M, Llesuy S, Gutierrez S, Repetto M. Peripheral parameters of oxidative stress in Graves' disease: the effects of methimazole and 131 iodine treatments. Clin Endocrinol (Oxf) 2003;59:321-327

12. Nordyke RA, Gilbert FI Jr. Optimal iodine-131 dose for eliminating hyperthyroidism in Graves' disease. J Nucl Med 1991;32:411-416

13. Sabri O, Zimny M, Schreckenberger M, Reinartz P, Nowak B, Ostwald E, et al. Characterization of therapy failures in radioiodine therapy of Graves' disease without simultaneous antithyroid agents. Nuklearmedizin 2001;40:1-6

14. Walter MA, Christ-Crain M, Muller B, Muller-Brand J. Radioiodine uptake and thyroid hormone levels on or off simultaneous carbimazole medication: a prospective paired comparison. Nuklearmedizin 2005;44:33-36

15. Toft AD. Clinical practice. Subclinical hyperthyroidism. N Engl J Med 2001;345:512-516

16. Walter MA, Christ-Crain M, Eckard B, Schindler C, Nitzsche EU, Muller-Brand J, et al. Radioiodine therapy in hyperthyroidism: inverse correlation of pretherapeutic iodine uptake level and post-therapeutic outcome. Eur J Clin Invest 2004;34:365-370

17. Bockisch A, Jamitzky T, Derwanz R, Biersack HJ. Optimized dose planning of radioiodine therapy of benign thyroidal diseases. J Nucl Med 1993;34:1632-1638 
18. Marinelli LD. Dosage determination in the use of radioactive isotopes. J Clin Invest 1949;28:1271-1280

19. Crooks J, Wayne EJ, Robb RA. A clinical method of assessing the results of therapy in thyrotoxicosis. Lancet 1960;1:397-401

20. Imseis RE, Vanmiddlesworth L, Massie JD, Bush AJ, Vanmiddlesworth NR. Pretreatment with propylthiouracil but not methimazole reduces the therapeutic efficacy of iodine-131 in hyperthyroidism. J Clin Endocrinol Metab 1998;83:685-687

21. Korber C, Schneider P, Korber-Hafner N, Hanscheid H, Reiners C. Antithyroid drugs as a factor influencing the outcome of radioiodine therapy in Graves' disease and toxic nodular goitre? Eur J Nucl Med 2001;28:1360-1364

22. Bonnema SJ, Bennedbaek FN, Veje A, Marving J, Hegedus L. Propylthiouracil before ${ }^{131} \mathrm{I}$ therapy of hyperthyroid diseases: effect on cure rate evaluated by a randomized clinical trial. J Clin Endocrinol Metab 2004;89:4439-4444

23. Hancock LD, Tuttle RM, LeMar H, Bauman J, Patience T. The effect of propylthiouracil on subsequent radioactive iodine therapy in Graves' disease. Clin Endocrinol (Oxf) 1997; $47: 425-430$
24. Tuttle RM, Patience T, Budd S. Treatment with propylthiouracil before radioactive iodine therapy is associated with a higher treatment failure rate than therapy with radioactive iodine alone in Graves' disease. Thyroid 1995;5:243-247

25. Santos RB, Romaldini JH, Ward LS. Propylthiouracil reduces the effectiveness of radioiodine treatment in hyperthyroid patients with Graves' disease. Thyroid 2004;14:525-530

26. Marcocci C, Gianchecchi D, Masini I, Golia F, Ceccarelli C, Bracci $\mathrm{E}$, et al. A reappraisal of the role of methimazole and other factors on the efficacy and outcome of radioiodine therapy of Graves' hyperthyroidism. J Endocrinol Invest 1990; $13: 513-520$

27. Koornstra JJ, Kerstens MN, Hoving J, Visscher KJ, Schade JH, Gort HB, et al. Clinical and biochemical changes following ${ }^{131} \mathrm{I}$ therapy for hyperthyroidism in patients not pretreated with antithyroid drugs. Neth J Med 1999;55:215-221 